\title{
Matrix Metalloproteinase-1 Inhibitory Activities of Morinda citrifolia Seed Extract and Its Constituents in UVA-Irradiated Human Dermal Fibroblasts
}

\author{
Megumi Masuda, ${ }^{a}$ Kazuya Murata, ${ }^{a}$ Shunsuke Naruto, ${ }^{a}$ Akemi Uwaya,${ }^{b}$ Fumiyuki Isami, ${ }^{b}$ and \\ Hideaki Matsuda*,a \\ ${ }^{a}$ Faculty of Pharmacy, Kinki University; 3-4-1 Kowakae, Higashiosaka, Osaka 577-8502, Japan: and ${ }^{b}$ Research and \\ Development, Tahitian Noni Inc.; 3-2-2 Nishishinjuku, Shinjuku-ku, Tokyo 160-0023, Japan. \\ Received September 20, 2011; accepted November 7, 2011; published online November 17, 2011
}

The objective of this study was to examine whether a $50 \%$ ethanolic extract (MCS-ext) of the seeds of Morinda citrifolia (noni) and its constituents have matrix metalloproteinase-1 (MMP-1) inhibitory activity in UVA-irradiated normal human dermal fibroblasts (NHDFs). The MCS-ext $(10 \mu \mathrm{g} / \mathrm{mL})$ inhibited MMP-1 secretion from UVA-irradiated NHDFs, without cytotoxic effects, at $48 \mathrm{~h}$ after UV exposure. The ethyl acetatesoluble fraction of MCS-ext was the most potent inhibitor of MMP-1 secretion. Among the constituents of the fraction, a lignan, 3,3'-bisdemethylpinoresinol (1), inhibited the MMP-1 secretion at a concentration of $0.3 \mu \mathrm{M}$ without cytotoxic effects. Furthermore, $1(0.3 \mu \mathrm{M})$ reduced the level of intracellular MMP-1 expression. Other constituents, namely americanin A (2), quercetin (3) and ursolic acid (4), were inactive. To elucidate inhibition mechanisms of MMP-1 expression and secretion, the effect of 1 on mitogen-activated protein kinases (MAPKs) phosphorylation was examined. Western blot analysis revealed that $1(0.3 \mu \mathrm{M})$ reduced the phosphorylations of $\mathbf{p 3 8}$ and c-Jun-N-terminal kinase (JNK). These results suggested that 1 suppresses intracellular MMP-1 expression, and consequent secretion from UVA-irradiated NHDFs, by down-regulation of MAPKs phosphorylation.

Key words Morinda citrifolia; noni seed; matrix metalloproteinase-1 inhibitor; photoaging; 3,3'-bisdemethylpinoresinol

Normal human dermis primarily consists of an extracellular matrix of connective tissue, of which the most abundant protein is collagen. Chronic UV exposure denatures collagen and elastic fibers in the dermis and induces wrinkles, thereby promoting photoaging. ${ }^{1)} \mathrm{UV}$ induces the production of matrix metalloproteinases (MMPs) by activating intracellular signal transcription pathways, including mitogen-activated protein kinase (MAPK) p38 and c-Jun-N-terminal kinase (JNK). ${ }^{2)}$ Activation of the phosphorylation of MAPKs may lead to an over expression of c-Jun and c-Fos. This results in induction of activator protein-1 (AP-1), which, in turn, up-regulates expression of MMPs. ${ }^{3)}$ MMPs are a family of structurally related matrix degrading enzymes that are associated with various destructive processes, including inflammation, tumor invasion and skin aging. ${ }^{1)}$ MMPs are subdivided in different types at least over 20 members. ${ }^{4}$ Among them, MMP-1, secreted from human skin fibroblasts, is mainly responsible for the degradation of dermal collagen in the photoaging process of human skin. ${ }^{5)}$ Neutrophils play an important role during the process of photoaging, which are set in motion by UV irradiation. The neutrophils infiltrate into the skin and release several active enzymes, such as MMPs and human leukocyte elastase (HLE). ${ }^{6}$ HLE cleaves the helix structure of type I collagen and then degrades elastic fiber in human skin. ${ }^{7)}$ HLE also directly activates MMP-1 from human dermal fibroblasts. ${ }^{8)}$ Thus, it is expected that MMP-1 inhibitors and/or HLE inhibitors may be useful for the prevention of photoaging of human skin.

Morinda citrifolia L. (Rubiaceae), commonly known as "noni" in Hawaii and Tahiti, is a tropical tree. The fruits, roots, bark and leaves of noni have been used throughout Polynesia as a folk medicine for the treatment of many diseases. ${ }^{9)}$ Recently, the noni fruit juice and tea made from noni leaves have been introduced to the functional food market. Noni fruit contains a large number of seeds throughout its flesh. During the production of noni fruit juice, these seeds are removed and discarded. Consequently, we have investigated the utility of noni seed and previously reported that a $50 \%$ ethanolic extract from noni seeds (MCS-ext) had more potent in vitro HLE inhibitory activity than the extracts of noni leaves or fruit flesh. ${ }^{10)}$ MCS-ext also exhibited tyrosinase inhibitory and 1,1-diphenyl-2-picrylhydrazyl (DPPH) radical scavenging activities. Activity-guided fractionation of MCSext led to the isolation of 3,3'-bisdemethylpinoresinol (1), americanin A (2) and quercetin (3) as active constituents with both tyrosinase inhibitory and DPPH radical scavenging activities. Ursolic acid (4) was isolated as a triterpenoid with potent HLE inhibitory activity. Compounds $\mathbf{2}$ and $\mathbf{3}$ also showed superoxide dismutase like activity. ${ }^{10)}$ If MCS-ext is proven to be MMP-1 inhibitor, an extract with HLE inhibitory activity may be an ideal ingredient for anti-photoaging cosmetics. The objective of this study was to determine if MCS-ext and its constituents have MMP-1 inhibitory activity in UVA-irradiated normal human dermal fibroblasts (NHDFs).

\section{MATERIALS AND METHODS}

Plant Materials and Reagents Fruits of $M$. citrifolia were collected in French Polynesia during 2004-2006. The sample was identified by Tropical Resources, Inc. (Provo, UT, U.S.A.). The fruits were separated into flesh and seeds by hand. The seeds were air-dried. Voucher specimens of dried seeds (Noni Seed: 004-239A-0014) are deposited at Kinki University. The preparation of $50 \%$ ethanolic extract of dried seed (MCS-ext, yield 5\%) and the isolation of lignans $(1,2)$ have been described in our previous paper. ${ }^{10)}$ Quercetin 
(3) and ursolic acid (4) were purchased from Wako Pure Chemical Industries, Ltd. (Osaka, Japan). Epigallocatechin-3$\mathrm{O}$-gallate (EGCG) was purchased from Sigma-Aldrich Co. (St. Louis, MO, U.S.A.). Other chemical and biochemical reagents were of reagent grade and were purchased from Wako Pure Chemical Industries, Ltd. and/or Nacalai Tesque, Inc. (Kyoto, Japan) unless otherwise noted.

Fractionation of MCS-ext A suspension of MCS-ext $(30 \mathrm{~g})$ in water $(120 \mathrm{~mL})$ was extracted with hexane $(120 \mathrm{~mL} \times 2)$ followed by ethyl acetate $(120 \mathrm{~mL} \times 10)$. Evaporation of solvent from each combined extract gave a hexane-soluble fraction $(0.29 \mathrm{~g}$; yield from MCS-ext: $1 \%)$ and an ethyl acetate-soluble fraction $(5.4 \mathrm{~g} ; 18 \%)$. The aqueous layer was evaporated and then lyophilized to give a water-soluble fraction ( $22 \mathrm{~g} ; 73 \%)$.

Cell Culture Normal human dermal fibroblasts (NHDFs) obtained from neonatal foreskins was purchased from Kurabo Industries, Ltd. (Osaka, Japan) in Feb. 2010. NHDFs were precultured in Dulbecco's modified Eagle's medium (DMEM) supplemented with $10 \%(\mathrm{v} / \mathrm{v})$ fetal bovine serum (Nichirei Biosciences Inc., Tokyo, Japan) at $37^{\circ} \mathrm{C}$ in a humidified $\mathrm{CO}_{2}-$ controled (5\%) incubator. NHDFs from passages 3 to 6 were used for the experiments.

Assay of Cell Viability The cell viability was determined by a 2-(2-methoxy-4-nitrophenyl)-3-(4-nitrophenyl)5-(2,4-disulfophenyl)-2H-tetrazolium (WST-8) assay using a commercial kit (Tetra Color ONE, Seikagaku Corporation, Tokyo, Japan). Test samples were dissolved in dimethyl sulfoxide (DMSO) and then diluted with DMEM to an appropriate concentration. In the control group, $0.2 \%(\mathrm{v} / \mathrm{v}) \mathrm{DMSO} /$ DMEM solution was used instead of sample solution. The final concentration of DMSO was $0.2 \%(\mathrm{v} / \mathrm{v})$. Briefly, $1 \times 10^{4}$ cells in the medium $(100 \mu \mathrm{L})$ were placed on 96-well microplate. After $24 \mathrm{~h}$ of incubation, several concentrations of samples in the medium $(100 \mu \mathrm{L})$ were added. After incubation for $48 \mathrm{~h}$, the medium was replaced with a new medium $(10 \%$ WST-8/DMEM, $100 \mu \mathrm{L})$. After incubation for $1 \mathrm{~h}$, the resulting formazan was photometrically determined at $450 \mathrm{~nm}$ with a microplate reader. Cell viability was expressed as a percentage of the control cells.

UV Irradiation NHDFs were cultured until confluent on 6-well microplate. The cells were washed twice with phosphate-buffered saline (PBS, pH 7.4) and exposed to UVA irradiation ( $365 \mathrm{~nm}$; FL20S•BLB/DMR, Toshiba Co., Tokyo, Japan) in PBS ( $1 \mathrm{~mL})$. The total energy dose of UVA irradiation was $5 \mathrm{~J} / \mathrm{cm}^{2}$. Using the trypan blue (Life Technologies Japan Ltd., Tokyo, Japan) exclusion test, the UV dose did not affect the cellular viability (data not shown). After UVA exposure, the cells were washed twice with PBS and the test sample solution $(2 \mathrm{~mL})$ was immediately added to each well. In the control and the vehicle control groups, $0.2 \%$ (v/v) DMSO/ DMEM solution was used instead of sample solution. In the vehicle control and test groups, NHDFs were exposed to UVA.

Western Blot Analysis for MMP-1 in Cultured Medium After irradiation, NHDFs were incubated at $37^{\circ} \mathrm{C}$ for various lengths of time in serum free medium on 6 -well microplate. Aliquots of the cultured NHDFs medium were centrifuged at $12000 \times \boldsymbol{g}$ for $15 \mathrm{~min}$ at $4^{\circ} \mathrm{C}$. The supernatant was boiled for $4 \mathrm{~min}$ in sodium dodecyl sulfate (SDS)containing sample buffer (Bio-Rad Laboratories, Hercules, CA, U.S.A.) containing $5 \%(\mathrm{v} / \mathrm{v}) \beta$-mercaptoethanol and then chilled on ice. An equal volume of resulting supernatant was separated by $10 \%$ SDS-polyacrylamide gel electrophoresis (SDS-PAGE). The separated proteins were transferred onto polyvinylidene fluoride (PVDF) membranes (Bio-Rad Laboratories). The membranes were subsequently blocked overnight at $4^{\circ} \mathrm{C}$ with $5 \%(\mathrm{w} / \mathrm{v})$ skim milk in Tris-buffered saline (TBS; $25 \mathrm{~mm}$ Tris, $137 \mathrm{~mm} \mathrm{NaCl}, 2.68 \mathrm{~mm} \mathrm{KCl}$ ) containing $0.1 \%$ Tween 20 and then incubated with anti-MMP-1 antibody (Santa Cruz Biotechnology Inc., Santa Cruz, CA, U.S.A.) at $1: 400$ dilution for $1 \mathrm{~h}$ at room temperature. Subsequently, the membranes were incubated with horseradish peroxidase-conjugated anti-goat immunoglobulin $\mathrm{G}$ ( $\mathrm{IgG}$ ) secondary antibody (Santa Cruz Biotechnology Inc.) at 1:4000 dilution for $1 \mathrm{~h}$ at room temperature. The resulting proteins were detected by enhanced chemiluminescence Western blot detection reagents kit (ECL Plus, GE Healthcare U.K. Ltd., Little Chalfont, Buckinghamshire, U.K.), and visualized with the ChemiDoc XRS Plus System (Bio-Rad Laboratories Inc.). Relative band density was determined by Quantity One 1-D Analysis Software (Version. 4.6.7, 2008; Bio-Rad Laboratories Inc.).

Western Blot Analysis for MAPKs and Intracellar MMP-1 The cultured NHDFs were washed twice with precooled PBS ( $\mathrm{pH}$ 7.4) and then lysed with a cell lysis buffer (Cell Signaling Technology, Danvers, MA, U.S.A.) containing $1 \mathrm{~mm}$ phenymethanesulfonyl fluoride. The lysates were centrifuged at $12000 \times \boldsymbol{g}$ for $15 \mathrm{~min}$ at $4^{\circ} \mathrm{C}$. The supernatant was subjected to Western blot analysis, as described above. Protein concentrations of samples were determined by DC protein assay reagent (Bio-Rad Laboratories Inc.) with bovine serum albumin (Bio-Rad Laboratories Inc.) as a standard. Equal amounts of samples were separated by $10 \%$ SDS-PAGE and transferred onto PVDF membranes. The membranes were subsequently blocked with $5 \%(\mathrm{w} / \mathrm{v})$ skim milk in TBS containing $0.1 \%$ Tween 20 or Blocking One-P overnight at $4^{\circ} \mathrm{C}$ and then incubated with the indicated antibodies. The antibody against MMP-1 (Santa Cruz Biotechnology Inc.) was used at 1:400 dilution. The membranes were exposed to horseradish peroxidase-conjugated anti-goat IgG secondary antibody (Santa Cruz Biotechnology Inc.) at 1:4000 dilution. Antibodies against p38, phospho-p38, JNK, phospho-JNK and $\beta$-actin were purchased from Cell Signaling Technology and used at 1:1000 dilution. Subsequently, the membranes were exposed to the horseradish peroxidase-conjugated anti-rabbit IgG secondary antibody (GE Healthcare U.K. Ltd.) at $1: 2000$ dilution. The resulting proteins were detected by ECL Plus, and analyzed by ChemiDoc XRS Plus System, as described above.

Statistical Analysis Each experiment was performed in triplicate unless otherwise noted. Intergroup differences were evaluated by one-way analysis of variance (ANOVA) followed by Dunnett's multiple comparison test. GraphPad Prism for Windows, Ver. 5 (GraphPad Software Inc., 2007) was used for the statistical analyses.t

\section{RESULTS}

Effect of MCS-ext on MMP-1 Secretion from UVAIrradiated NHDFs MMP-1 protein in the culture medium was assessed by Western blot analysis. NHDFs were exposed to UVA at a dose of $5 \mathrm{~J} / \mathrm{cm}^{2}$. UVA irradiation time-dependently enhanced the secretion of MMP-1 from NHDFs in the vehicle control group at 9 to $48 \mathrm{~h}$, as compared to the con- 


\begin{tabular}{|c|c|c|c|c|c|c|c|c|}
\hline \multirow{3}{*}{$\begin{array}{r}\text { Time (h) } \\
\text { MCS-ext }(\mu \mathrm{g} / \mathrm{mL})\end{array}$} & \multicolumn{3}{|c|}{ UVA } & \multicolumn{2}{|r|}{ UVA } & \multicolumn{3}{|c|}{ UVA } \\
\hline & \multicolumn{3}{|c|}{9} & \multicolumn{2}{|r|}{24} & \multicolumn{3}{|c|}{48} \\
\hline & 0 & 0 & 10 & 0 & $\begin{array}{ll}0 & 10\end{array}$ & 0 & 0 & 10 \\
\hline & Control & $\begin{array}{l}\text { Vehicl } \\
\text { contro }\end{array}$ & & Contr & $\begin{array}{l}\text { Vehicle } \\
\text { control }\end{array}$ & Control & $\begin{array}{l}\text { Jehic } \\
\text { contrc }\end{array}$ & \\
\hline MMP-1 & 20 & & & $=$ & $\rightarrow$ & & & \\
\hline
\end{tabular}

Fig. 1. Effect of MCS-ext on MMP-1 Secretion from UVA-Irradiated NHDFs

Fibroblasts were exposed to UVA $\left(5 \mathrm{~J} / \mathrm{cm}^{2}\right)$ and then cultured in the serum free medium containing test samples for 9,24 and $48 \mathrm{~h}$. MMP-1 protein levels in the cultured medium at the indicated times were assessed by Western blot analysis using the antibody against human MMP-1. The blot is representative of three separate experiments and represents a single immunoblot.

\begin{tabular}{|c|c|c|c|c|c|c|}
\hline & \multirow[b]{3}{*}{0} & \multicolumn{5}{|c|}{ UVA } \\
\hline & & & $\begin{array}{l}\text { Hexane } \\
\text { soluble- } \\
\text { fraction }\end{array}$ & $\begin{array}{l}\text { Ethyl acetate } \\
\text { soluble- } \\
\text { fraction }\end{array}$ & $\begin{array}{l}\text { e Water } \\
\text { soluble- } \\
\text { fraction }\end{array}$ & EGCG \\
\hline & & 0 & 3 & $\begin{array}{c}3 \\
(\mu \mathrm{g} / \mathrm{mL})\end{array}$ & 3 & $\begin{array}{c}0.01 \underset{(\mu \mathrm{M})}{0.1} \\
\end{array}$ \\
\hline MMP-1 & 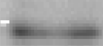 & 0 & $1=$ & $=-1=$ & $=1$ & $-0=$ \\
\hline
\end{tabular}

Fig. 2. Effects of Hexane-, Ethyl Acetate- and Water-Soluble Fractions from MCS-ext and EGCG on MMP-1 Secretion from UVA-Irradiated NHDFs

Fibroblasts were exposed to UVA $\left(5 \mathrm{~J} / \mathrm{cm}^{2}\right)$ and then cultured in the serum free medium containing test samples for $48 \mathrm{~h}$. MMP-1 protein levels in the cultured medium were assessed by Western blot analysis using the antibody against human MMP-1. The blot is representative of three separated experiments and represents a single immunoblot. EGCG (epigallocatechin-3-O-gallate) was used as a reference compound.

trol group without UVA irradiation (Fig. 1). UVA-irradiated NHDFs were treated with MCS-ext $(10 \mu \mathrm{g} / \mathrm{mL})$ immediately after UVA exposure. MCS-ext obviously inhibited the secretion of MMP-1 from UVA-irradiated NHDFs at 9 to $48 \mathrm{~h}$. MCS-ext had no cytotoxic effect at 3 to $30 \mu \mathrm{g} / \mathrm{mL}$ (cell viability: $98 \pm 2 \%, 98 \pm 6,99 \pm 4 \%$ at $3,10,30 \mu \mathrm{g} / \mathrm{mL}$, respectively). Thus, we assessed the level of MMP-1 secretion into the culture medium at $48 \mathrm{~h}$ when the inhibition of MMP-1 secretion was most distinctly detected.

Effects of Hexane-, Ethyl Acetate- and Water-Soluble Fractions Obtained from MCS-ext and EGCG on MMP-1 Secretion from UVA-Irradiated NHDFs Fractionation of MCS-ext by successive solvent extraction gave a hexane-, an ethyl acetate- and a water-soluble fraction. Effects of these fractions and EGCG, a well known inhibitor of MMP-1 secretion, ${ }^{11)}$ on MMP-1 secretion from UVA-irradiated NHDFs were assessed. Results of Western blot analysis are shown in Fig. 2. EGCG showed potent inhibition of MMP-1 secretion at a concentration of $0.1 \mu \mathrm{M}$. Although all three fractions $(3 \mu \mathrm{g} /$ $\mathrm{mL}$ ) significantly inhibited the MMP-1 secretion at $48 \mathrm{~h}$ compared to the vehicle control group, the ethyl acetate-soluble fraction showed the most potent inhibition, but had no cytotoxic effect at 1 to $10 \mu \mathrm{g} / \mathrm{mL}$ (cell viability: $102 \pm 11,98 \pm 5$, $102 \pm 5 \%$ at $1,3,10 \mu \mathrm{g} / \mathrm{mL}$, respectively).

Effects of Constituents of MCS-ext and EGCG on MMP-1 Secretion from UVA-Irradiated NHDFs As reported in our previous paper, ${ }^{10}$ 3,3'-bisdemethylpinoresinol (1, isolation yield from MCS-ext: $1.17 \%$; isolation yield from dried seeds: $0.065 \%)$, americanin A $(2,0.51 \%$; $0.024 \%)$, quercetin $(3,0.19 \% ; 0.009 \%)$ and ursolic acid $(4,0.74 \% ; 0.035 \%)$ have been isolated from the ethyl acetate-soluble fraction. The effects of these constituents on MMP-1 secretion were examined by Western blot analysis, and the relative intensity of each blot shown in Fig. 3Aa was measured. The serum free medium containing each constituent at a concentration of $0.03,0.1$ and $0.3 \mu \mathrm{M}$ was added to UVA-irradiated NHDFs im- mediately after UVA exposure, and the mixture was incubated for $48 \mathrm{~h}$. As shown in Figs. 3Aa and Ab, $1(0.3 \mu \mathrm{M})$ obviously reduced the levels of MMP-1 secretion, compared to the vehicle control group. On the other hand, 2 (Fig. 3Ba), 3 (Fig. $3 \mathrm{Bb}$ ) and 4 (Fig. $3 \mathrm{Bc}$ ) were inactive at $0.3 \mu \mathrm{m}$. In the range of 0.03 to $0.3 \mu \mathrm{M}, \mathbf{1}, \mathbf{2}, \mathbf{3}$ and $\mathbf{4}$ had no cytotoxic effect, as shown in Fig. 3C.

Effects of 3,3'-Bisdemethylpinoresinol (1) on Intracellular MMP-1 Expression in UVA-Irradiated NHDFs To elucidate the mechanism of MMP-1 inhibition, we examined the effect of 1 on intracellular MMP-1 expression in UVAirradiated NHDFs, as well as MMP-1 secretion into the culture medium. The time-course of intracellular MMP-1 expression and MMP-1 secretion after UVA-irradiation is shown in Fig. 4. In the vehicle control group, UVA irradiation gradually enhanced the levels of intracellular MMP-1 expression during 24 to $48 \mathrm{~h}$. The most distinct MMP-1 expression was detected at $48 \mathrm{~h}$, as shown in Fig. 4A. Compound $1(0.3 \mu \mathrm{M})$ obviously inhibited the increase of intracellular MMP-1 expression at $48 \mathrm{~h}$. The levels of MMP-1 secretion into the cultured medium from UVA-irradiated NHDFs were increased at $48 \mathrm{~h}$ in the vehicle control group, whereas the treatment of the cells with 1 obviously inhibited MMP-1 secretion (Fig. 4B).

Effect of UVA Irradiation on the Levels of MAPKs Phosphorylation in NHDFs To further understand the mechanism of MMP-1 inhibition in NHDFs by $\mathbf{1}$, the activation of MAPKs after exposure to UVA irradiation ( $5 \mathrm{~J} /$ $\mathrm{cm}^{2}$ ) was preliminarily examined by Western blot analysis. The time-courses of JNK and p38 phosphorylation levels are shown in Fig. 5A. UVA irradiation resulted in a rapid and transient activation of JNK phosphorylation at 0.5 to $1.0 \mathrm{~h}$ after exposure. The p38 phosphorylation levels were remarkably enhanced in a rapid and transient manner at 0 to $1.0 \mathrm{~h}$ after exposure. The basal levels of JNK and p38 in NHDFs were not affected by UVA irradiation. 
A a

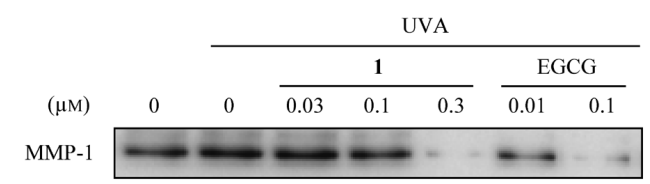

b

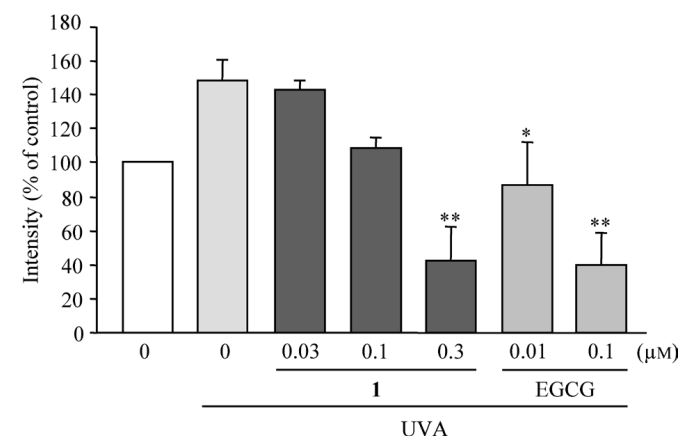

Fig. 3A. Effects of 3,3'-Bisdemethylpinoresinol (1) and EGCG on MMP-1 Secretion from UVA-Irradiated NHDFs

Fibroblasts were exposed to UVA $\left(5 \mathrm{~J} / \mathrm{cm}^{2}\right)$ and then cultured in the serum free medium containing test samples for $48 \mathrm{~h}$. MMP-1 protein levels in the cultured medium were assessed by Western blot analysis using the antibody against human MMP-1. (a) The blot is representative of three separated experiments and represents a single immunoblot. (b) All data are reported as mean \pm S.E. of three separate experiments. ${ }^{*} p<0.05$ and $* * p<0.01$ : statistically significant $v s$. the value of vehicle control group.

$\mathrm{B}$

a

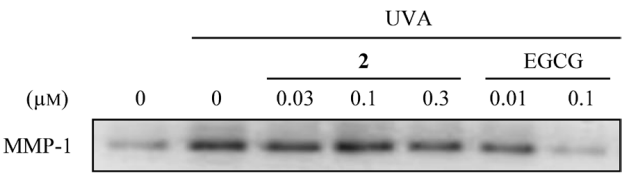

b

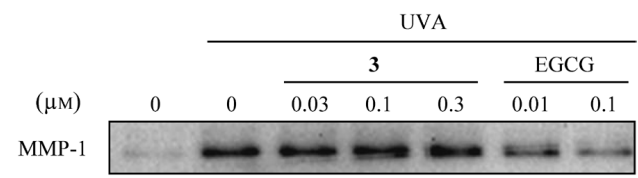

C

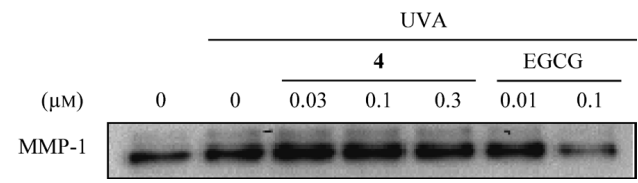

Fig. 3B. Effects of Americanin A (2), Quercetin (3), and Ursolic Acid (4) and EGCG on MMP-1 Secretion from UVA-Irradiated NHDFs

Fibroblasts were exposed to UVA $\left(5 \mathrm{~J} / \mathrm{cm}^{2}\right)$ and then cultured in the serum free medium containing test samples for $48 \mathrm{~h}$. MMP-1 protein levels in the cultured medium were assessed by Western blot analysis using the antibody against human MMP-1. The blot is representative of three separate experiments and represents a single immunoblot.

$\mathrm{C}$

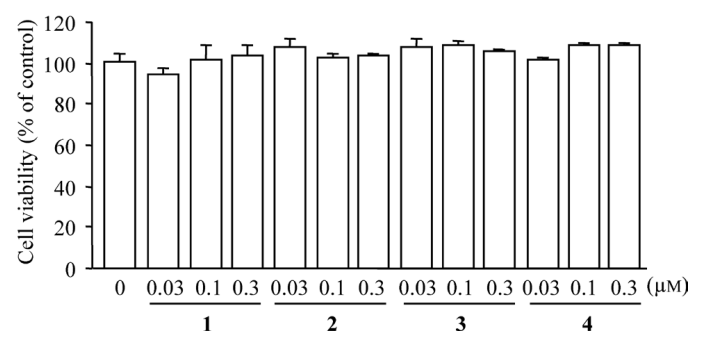

Fig. 3C. Effects of 3,3'-Bisdemethylpinoresinol (1), Americanin A (2), Quercetin (3) and Ursolic Acid (4) on Cell Viability

Fibroblasts were incubated in serum free cultured medium in the presence of the indicated concentration of samples for $48 \mathrm{~h}$. Cell viability was determined by a WST- 8 assay. All date was given as mean \pm S.E. of three separated experiments.
Effect of 3,3'-Bisdemethylpinoresinol (1) on the Levels of MAPKs Phosphorylation in UVA-Irradiated NHDFs Effect of $\mathbf{1}(0.1,0.3 \mu \mathrm{M})$ on JNK and p38 phosphorylation in UVA-irradiated NHDFs was examined. On the basis of above preliminary results (Fig. 5A), phosphorylation levels were assessed at $0.5 \mathrm{~h}$ after UVA-irradiation. As shown in Fig. $5 \mathrm{~B}, 1(0.3 \mu \mathrm{M})$ obviously inhibited the phosphorylation of both JNK and p38 at $0.5 \mathrm{~h}$ after, whereas a lower concentration of $\mathbf{1}$ $(0.1 \mu \mathrm{M})$ produced no significant inhibition.

\section{DISCUSSION}

MMP-1 is a major collagenolytic enzyme responsible for collagen damage in UV-irradiated human skin. ${ }^{12)}$ It has been demonstrated that UV irradiation enhances MMP-1 expression in $\mathrm{NHDFs}^{13)}$ and increases MMP-1 secretion into culture medium. ${ }^{14)}$ In the past decade, a number of papers have reported the isolation of MMP-1 inhibitors from plant resources. Examples include aucubin from Eucommia ulmoides cortex, ${ }^{15)}$ tectoridin-4'- $O-\beta$-D-glucoside from aerial parts of Viola hondoensis, ${ }^{16)}$ sumaflavone and amentoflavone from whole plant of Selaginella tamariscina, ${ }^{17)}$ styraxjaponoside B and erythrodiol-3-acetate from Styrax japonica stem, ${ }^{18,19)}$ xanthorrhizol from Curcuma xanthorrhiza rhizome, ${ }^{14)}$ caffeic acid and chlorogenic acid from Coffea arabica leaf, ${ }^{20)}$ panduratin $\mathrm{A}$ and 4-hydroxypanduratin A from Kaempferia pandurata rhizome, ${ }^{21,22)}$ Punica granatum extract, ${ }^{23)}$ Emblica officinalis extract $^{24)}$ and Sanguisorba officinalis extract. ${ }^{25)}$ Among these plants, a few plant such as Coffea arabica leaf extract ${ }^{20)}$ is known to have HLE inhibitory activity. It has been demonstrated that MCS-ext exhibits HLE inhibitory activity. ${ }^{10)}$ HLE inhibitors with MMP-1 inhibitory activity are ideal ingredients for anti-photoaging cosmetics. Therefore, as part of our continuing investigation of noni seeds, we examined MCSext for MMP-1 inhibitory activity. UVB-irradiated NHDFs were used to assay MMP-1 inhibitory activity of almost all constituents of the plants cited above. Further, UVA-induced photo-damage of the skin is believed to be the result of effects on dermal fibroblasts by deeply penetrating UVA radiation, as opposed to UVB radiation which only penetrates the epidermis. ${ }^{13)}$ Therefore, we used UVA-irradiated NHDFs for the evaluation of MMP-1 inhibitory activity. UVA irradiation was performed at $5 \mathrm{~J} / \mathrm{cm}^{2}$, according to a previously reported method $^{26)}$ in which the UV dose enhanced MMP-1 secretion without any significant effects on cell viability.

The MCS-ext significantly reduced the secretion of MMP-1 without any cytotoxic effects. Activity guided fractionation of MCS-ext was carried out to identify the active constituents of MCS-ext by successive solvent extractions. The ethyl acetatesoluble fraction exhibited the most potent activity. Therefore, we focused on the fraction from which two lignans $(\mathbf{1}, \mathbf{2}), 3$ and 4 were previously isolated as active constituents having potent DPPH radical scavenging, tyrosinase inhibitory and HLE inhibitory activities. ${ }^{10)}$ The MMP-1 inhibitory activities of $\mathbf{1}, \mathbf{2}, \mathbf{3}$ and $\mathbf{4}$ were assayed. The lignan (1) concentrationdependently displayed potent inhibitory activity without any significant effects on cell viability.

Compound 3 had no inhibitory activity at $0.3 \mu \mathrm{M}$. But the effect of 3 on MMP-1 is controversial. Leu et al. ${ }^{27)}$ reported that $3(50 \mu \mathrm{g} / \mathrm{mL}, 166 \mu \mathrm{M})$ inhibited MMP-1 secretion from NHDFs at $24 \mathrm{~h}$. In addition, $\mathrm{Lim}$ and $\mathrm{Kim}^{28)}$ reported that 3 
UVA

\begin{tabular}{|c|c|c|c|c|c|c|c|c|}
\hline \multirow{2}{*}{$\begin{array}{r}\text { Time (h) } \\
1(\mu \mathrm{M})\end{array}$} & \multicolumn{2}{|c|}{4} & \multicolumn{2}{|c|}{8} & \multicolumn{2}{|c|}{24} & \multicolumn{2}{|c|}{48} \\
\hline & 0 & 0.3 & 0 & 0.3 & 0 & 0.3 & 0 & 0.3 \\
\hline Intracellular MMP-1 & & & & & & & + & 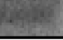 \\
\hline$\beta$-actin & & & & & & & & \\
\hline MMP-1 & & & & & $=$ & & En & $=$ \\
\hline
\end{tabular}

Fig. 4. (A) Effect of 3,3'-Bisdemethylpinoresinol (1) on the Intracellular MMP-1 Expression in UVA-Irradiated NHDFs, (B) Effect of 3,3'-Bisdemethylpinoresinol (1) on MMP-1 Secretion from UVA-Irradiated NHDFs

(A) Fibroblasts were exposed to UVA $\left(5 \mathrm{~J} / \mathrm{cm}^{2}\right)$ and then cultured in the serum free medium containing test samples for $4,8,24$ and $48 \mathrm{~h}$. Intracellular MMP-1 protein levels at the indicated times were assessed by Western blot analysis using the antibody against human MMP-1. The blot is representative of two separate experiments and represents a single immunoblot.

(B) Fibroblasts were exposed to UVA $\left(5 \mathrm{~J} / \mathrm{cm}^{2}\right)$ and then cultured in the serum free medium containing test samples for 24 to $48 \mathrm{~h}$. MMP-1 protein levels in cultured medium were assessed by Western blot analysis using the antibody against human MMP-1. The blot is representative of two separate experiments and represents a single immunoblot.

A

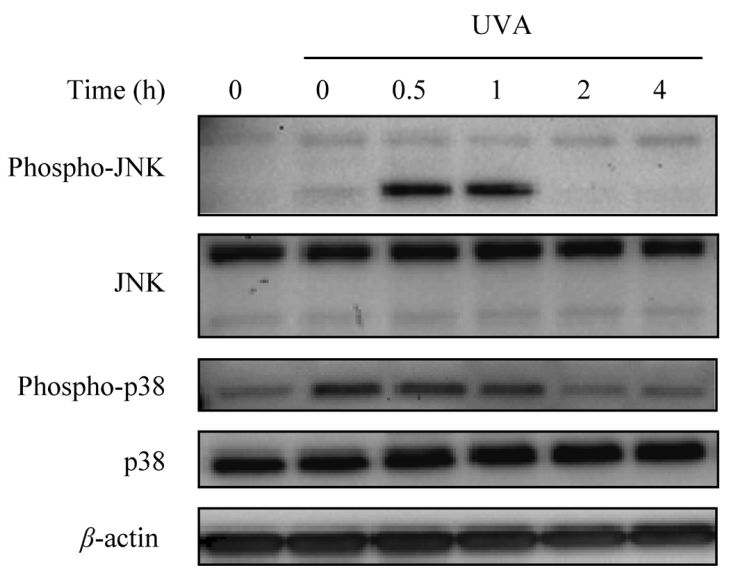

$\mathrm{B}$

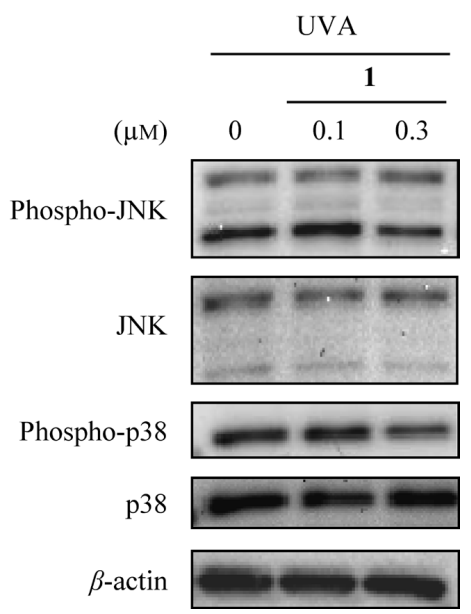

Fig. 5. (A) Time Course of the Phosphorylation Levels of MAPKs in UVA-Irradiated NHDFs, (B) Effect of 3,3'-Bisdemethylpinoresinol (1) on the Levels of MAPKs Phosphorylation in UVA-Irradiated NHDFs

(A) Fibroblasts were exposed to UVA $\left(5 \mathrm{~J} / \mathrm{cm}^{2}\right)$ and then cultured in the serum free medium for the indicated time. Total cellular proteins were prepared for Western blot analysis of MAPKs and phospho-MAPKs proteins using the antibodies against phospho-form and total-form of JNK and p38. $\beta$-Actin was used as an internal control. The blot is representative of three separate experiments and represents a single immunoblot.

(B) Fibroblasts were exposed to UVA $\left(5 \mathrm{~J} / \mathrm{cm}^{2}\right)$ and then cultured in the serum free medium for $0.5 \mathrm{~h}$. Total cellular proteins were prepared for Western blot analysis of MAPKs and phospho-MAPKs proteins using the antibodies against phospho-form and total-form of JNK and p38. $\beta$-Actin was used for internal control. The blot is representative of three separate experiments and represents a single immunoblot.

$(25 \mu \mathrm{M})$ down-regulated MMP-1 expression and secretion in 12- $O$-tetradecanoylphorbol 13 -acetate-treated NHDFs at $24 \mathrm{~h}$, but showed no effect at a concentration of $2.5 \mu \mathrm{M}$. In contrast, Cho et al. ${ }^{29)}$ recently described that $3(20,40 \mu \mathrm{M})$ enhanced MMP-1 expression in NHDFs at $24 \mathrm{~h}$, but was inactive at a concentration of $10 \mu \mathrm{M}$. Considering the content of $\mathbf{3}$ in MCSext, we used a dose of $3(0.03$ to $0.3 \mu \mathrm{M})$ in the experiments. Our inactive result of 3 at a low concentration $(0.3 \mu \mathrm{M})$ was not contradictory to the reported data. ${ }^{28,29)}$ However, there remained a discrepancy of the effect of $\mathbf{3}$ at high concentration (20 to $166 \mu \mathrm{M}$ ) on MMP-1 expression. Although we assumed that a part of the discrepancy might be due to the differences among several experimental conditions, further studies are required to prove the precise effect of $\mathbf{3}$ on MMP-1 in NHDFs.

Furthermore, 1 significantly inhibited the increase of intracellular MMP-1 expression, and decreased the level of MMP-1 secretion (Fig. 4). These results indicated that the inhibition of MMP-1 secretion by 1 was due to down-regulation of MMP-1 expression. Considering the content of $\mathbf{1}$ in MCS-ext, at least a portion of MMP-1 inhibitory activity of the extract may be attributed to $\mathbf{1}$.

Since $\mathbf{1}$ is newly described as a MMP-1 inhibitor, we attempted to elucidate its MMP-1 suppression mechanism. MAPKs signaling plays an important role in various cellular process, including MMP-1 secretion. ${ }^{5)} \mathrm{Klotz}$ et al. ${ }^{30)}$ reported that UVA radiation in human skin fibroblasts induces a pattern of MAPKs activation consisting of rapid and transcription induction of p38 and JNK activity but not extracellular signaling-regulated kinase. UVA irradiation $\left(5 \mathrm{~J} / \mathrm{cm}^{2}\right)$ to NHDFs resulted in a rapid and transient activation of the JNK phosphorylation. The level of p38 phosphorylation was remarkably enhanced in a more rapid and transient manner. These results (Fig. 5A) are in agreement with the reported data. ${ }^{30)} \mathrm{We}$ examined the effect of $\mathbf{1}$ on phosphorylation levels of MAPKs. The results (Fig. 5B) suggest that $\mathbf{1}$ may decrease JNK and p38 phosphorylation and suppress c-Fos and c-Jun expression, 
followed by inhibition of AP-1 and MMP-1 expression, as well as MMP-1 secretion.

In conclusion, MCS-ext significantly inhibited MMP-1 secretion from UVA-irradiated NHDFs. The lignan (1) was isolated from MCS-ext as an active constituent. Western blot analysis suggested that $\mathbf{1}$ inhibited the secretion of MMP-1 through reduction of $\mathrm{JNK}$ and p38 phosphorylation and intracellular MMP-1 expression. This is the first report of the MMP-1 inhibitory activity of noni. Since MCS-ext has both MMP-1 inhibitory and HLE inhibitory activities, the extract may be useful for the prevention of skin photoaging.

Acknowledgments This study was financially supported by the "Antiaging Center Project" for Private Universities from the Ministry of Education, Culture, Sports, Science and Technology of Japan, 2008-2012.

\section{REFERENCES}

1) Yaar M, Gilchrest BA. Photoageing: mechanism, prevention and therapy. Br. J. Dermatol., 157, 874-887 (2007).

2) Fisher GJ, Talwar HS, Lin J, Lin P, McPhillips F, Wang Z, Li X, Wan Y, Kang S, Voorhees JJ. Retinoic acid inhibits induction of cJun protein by ultraviolet radiation that occurs subsequent to activation of mitogen-activated protein kinase pathways in human skin in vivo. J. Clin. Invest., 101, 1432-1440 (1998).

3) Rittié L, Fisher GJ. UV-light-induced signal cascades and skin aging. Ageing Res. Rev., 1, 705-720 (2002).

4) Grant GM, Giambernardi TA, Grant AM, Klebe RJ. Overview of expression of matrix metalloproteinases (MMP-17, MMP-18, and MMP-20) in cultured human cells. Matrix Biol., 18, 145-148 (1999).

5) Wang $X Y, B i \quad Z G$. UVB-irradiated human keratinocytes and interleukin-1 $\alpha$ indirectly increase MAP kinase/AP-1 activation and MMP-1 production in UVA-irradiated dermal fibroblasts. Chin. Med. J., 119, 827-831 (2006).

6) Rijken F, Kiekens RCM, Bruijnzeel PLB. Skin-infiltrating neutrophils following exposure to solar-simulated radiation could play an important role in photoageing of human skin. Br. J. Dermatol., 152, $321-328$ (2005).

7) Kafienah W, Buttle DJ, Burnett D, Hollander AP. Cleavage of native type I collagen by human neutrophil elastase. Biochem. J., 330, 897-902 (1998).

8) Takeuchi H, Gomi T, Shishido M, Watanabe H, Suenobu N. Neutrophil elastase contributes to extracellular matrix damage induced by chronic low-dose UV irradiation in a hairless mouse photoaging model. J. Dermatol. Sci., 60, 151-158 (2010).

9) Ross IA. Medicinal Plants of the World, Vol. II, Chap. 16, Humana Press, Totowa, pp. 309-317 (2001).

10) Masuda M, Murata K, Fukuhama A, Naruto S, Fujita T, Uwaya A, Isami F, Matsuda H. Inhibitory effects of constituents of Morinda citrifolia seeds on elastase and tyrosinase. J. Nat. Med., 63, 267273 (2009).

11) Lee JH, Chung JH, Cho KH. The effects of epigallocatechin3-gallate on extracellular matrix metabolism. J. Dermatol. Sci., 40, 195-204 (2005)

12) Brennan M, Bhatti H, Nerusu KC, Bhagavathula N, Kang S, Fisher GJ, Varani J, Voorhees JJ. Matrix metalloproteinase-1 is the major collagenolytic enzyme responsible for collagen damage in UVirradiated human skin. Photochem. Photobiol., 78, 43-48 (2003).

13) Suganuma K, Nakajima H, Ohtsuki M, Imokawa G. Astaxanthin attenuates the UVA-induced up-regulation of matrix-metalloproteinase-1 and skin fibroblast elastase in human dermal fibroblasts. $J$. Dermatol. Sci., 58, 136-142 (2010).
14) Oh HI, Shim JS, Gwon SH, Kwon HJ, Hwang JK. The effect of xanthorrhizol on the expression of matrix metalloproteinase-1 and type-I procollagen in ultraviolet-irradiated human skin fibroblasts. Phytother. Res., 23, 1299-1302 (2009).

15) Ho JN, Lee YH, Lee YD, Jun WJ, Kim HK, Hong BS, Shin DH, Cho HY. Inhibitory effect of Aucubin isolated from Eucommia ulmoides against UVB-induced matrix metalloproteinase-1 production in human skin fibroblasts. Biosci. Biotechnol. Biochem., 69, 2227-2231 (2005).

16) Moon HI, Lee J, Zee OP, Chung JH. A glycosidic isoflavonoid from Viola hondoensis W. BECKER et $\mathrm{H}$. BoIsSieu (Violaceae), and its effect on the expression of matrix metalloproteinase-1 caused by ultraviolet irradiation in cultured human skin fibroblasts. Biol. Pharm. Bull., 28, 1123-1125 (2005).

17) Lee CW, Choi HJ, Kim HS, Kim DH, Chang IS, Moon HT, Lee SY, Oh WK, Woo ER. Biflavonoids isolated from Selaginella tamariscina regulate the expression of matrix metalloproteinase in human skin fibroblasts. Bioorg. Med. Chem., 16, $732-738$ (2008).

18) Kim MR, Moon HI, Chung JH, Moon YH, Hahm KS, Woo ER. Matrix metalloproteinase-1 inhibitor from the stem bark of Styrax japonica S. et Z. Chem. Pharm. Bull., 52, 1466-1469 (2004).

19) Moon HI, Lee J, Chung JH. The effect of erythrodiol-3-acetate on the expressions of matrix metalloproteinase-1 and type-1 procollagen caused by ultraviolet irradiated cultured primary old aged human skin fibroblasts. Phytomedicine, 13, 707-711 (2006).

20) Chiang HM, Lin TJ, Chiu CY, Chang CW, Hsu KC, Fan PC, Wen KC. Coffea arabica extract and its constituents prevent photoaging by suppressing MMPs expression and MAP kinase pathway. Food Chem. Toxicol., 49, 309-318 (2011).

21) Shim JS, Kwon YY, Han YS, Hwang JK. Inhibitory effect of panduratin A on UV-induced activation of mitogen-activated protein kinases (MAPKs) in dermal fibroblast cells. Planta Med., 74, 1446-1450 (2008).

22) Shim JS, Han YS, Hwang JK. The effect of 4-hydroxypanduratin A on the mitogen-activated protein kinase-dependent activation of matrix metalloproteinase-1 expression in human skin fibroblasts. $J$. Dermatol. Sci., 53, 129-134 (2009).

23) Aslam MN, Lansky EP, Varani J. Pomegranate as a cosmeceutical source: pomegranate fractions promote proliferation and procollagen synthesis and inhibit matrix metalloproteinase-1 production in human skin cells. J. Ethnopharmacol., 103, 311-318 (2006).

24) Fujii $T$, Wakaizumi M, Ikami $T$, Saito M. Amla (Emblica officinalis GAERTN.) extract promotes procollagen production and inhibits matrix metalloproteinase-1 in human skin fibroblasts. $J$. Ethnopharmacol., 119, 53-57 (2008).

25) Kim YH, Chung CB, Kim JG, Ko KI, Park SH, Kim JH, Eom SY, Kim YS, Hwang YI, Kim KH. Anti-wrinkle activity of ziyuglycoside I isolated from a Sanguisorba officinalis root extract and its application as a cosmeceutical ingredient. Biosci. Biotechnol. Biochem., 72, 303-311 (2008).

26) Fujimori H, Hisama M, Shibayama H, Iwaki M. Protecting effect of phytoncide solution, on normal human dermal fibroblasts against reactive oxygen species. J. Oleo Sci., 58, 429-436 (2009).

27) Leu SJ, Lin YP, Lin RD, Wen CL, Cheng KT, Hsu FL, Lee MH. Phenolic constituents of Malus doumeri var. formosana in the field of skin care. Biol. Pharm. Bull., 29, 740-745 (2006).

28) Lim H, Kim HP. Inhibition of mammalian collagenase, matrix metalloproteinase-1, by naturally-occurring flavonoids. Planta Med., 73, 1267-1274 (2007).

29) Cho JW, Cho SY, Lee SR, Lee KS. Onion extract and quercetin induce matrix metalloproteinase-1 in vitro and in vivo. Int. J. Mol. Med., 25, 347-352 (2010).

30) Klotz LO, Pellieux C, Briviba K, Pierlot C, Aubry JM, Sies H. Mitogen-activated protein kinase (p38-, JNK-, ERK-) activation pattern induced by extracellular and intracellular singlet oxygen and UVA. Eur. J. Biochem., 260, 917-922 (1999). 